\title{
Quantification of the uncertainty of pattern recognition approaches applied to acoustic emission signals
}

\author{
MARKUS G. R. SAUSE ${ }^{1}$ and SIEGFRIED HORN ${ }^{1}$ \\ 'University of Augsburg, Institute for Physics, Experimental Physics II, D-86135 \\ Augsburg \\ +498215983238 \\ $+498215983411$ \\ markus.sause@physik.uni-augsburg.de
}

Pattern recognition, cluster validity indices, acoustic emission, carbon fiber reinforced plastics

CFRP Carbon fiber reinforced plastics

$\mathrm{AE}$

Acoustic emission

$\mathrm{S}_{\mathrm{i}}$

Lamb-wave mode, symmetric, i-th order

$\mathrm{A}_{\mathrm{i}}$

Lamb-wave mode, antisymmetric, i-th order

$\mathrm{SH}_{\mathrm{i}}$

Shear-horizontal wave mode, i-th order

$\mathrm{C}_{\mathrm{ij}}$

Elastic coefficients

DB

Davies-Bouldin Index

TOU

Tou Index

S

Rousseuw's silhouette value

$\gamma$

Hubert's Gamma statistics

DCB

Double Cantilever Beam

$\mathrm{J}$

Degree of cluster separation

RAND

Rand Index

$\mathrm{C}$

Cluster validity measure

$\mathrm{C}_{0}$

Fit parameter (logistics function)

Fit parameter (logistics function)

$\mathrm{h}$

Fit parameter (logistics function)

$\mathrm{A}_{\min }$

Fit parameter (logistics function)

$\mathrm{A}_{\max }$

Fit parameter (logistics function)

A

Range of univariate distribution

$\mathrm{N}$

Univariate distribution 


\begin{abstract}
Acoustic emission analysis is a nondestructive technique frequently used to assess the integrity of fiber reinforced plastics. Pattern recognition techniques have shown great potential to identify microscopic failure mechanisms in plate-like structures. Because every assignment of an acoustic emission signal to a respective failure mechanism is possibly associated with an error, one key question is the reliability of the assignment method. It is useful to distinguish between the uncertainty of the assignment and the false assignment of an acoustic emission signal to a group of signals. The first is owed to statistical effects and the reliability of the classification method itself. The second is caused by false conclusions or disputable assumptions on the source mechanisms. The present study will focus on the first aspect. For this purpose, we propose a model based algorithm that estimates the uncertainty of a feature based pattern recognition approach based on cluster validity indices. Further, we demonstrate the application of the algorithm to experimental acoustic emission data obtained from a double cantilever beam specimens with unidirectional layup of carbon fiber reinforced polymer. Based on previous investigation we use a pattern recognition approach to distinguish between different failure mechanisms like matrix cracking, interfacial failure and fiber breakage based on the frequency features of the acoustic emission signals. We consider the influence of dispersion and attenuation effects during propagation of Lamb-waves on the extracted acoustic emission features. This is done by investigating the influence of source-sensor distance by test sources like pencil lead breaks and piezoelectric pulsers. Using the model based algorithm it is possible to calculate the uncertainty of the pattern recognition results as a function of source-sensor distance. It is found that dispersion effects of Lamb-waves do not seriously affect the distinction between microscopic failure mechanisms for source-sensor distances up to $375 \mathrm{~mm}$. We demonstrate that the spatial distribution of acoustic emission sources has a larger impact on the uncertainty of assignment than the absolute sourcesensor distance. Applying the proposed algorithm to the current experimental setup, we obtain an uncertainty of classification below $7 \%$ for source-sensor distances below $375 \mathrm{~mm}$. Attenuation is quantified to be $0.165 \mathrm{~dB} / \mathrm{mm}$ for the $\mathrm{A}_{0}$-mode and $0.047 \mathrm{~dB} / \mathrm{mm}$ for the $\mathrm{S}_{0}$-mode. Within the source-sensor distance of $375 \mathrm{~mm}$ this causes severe attenuation of the signal amplitude and thus prohibits detection of weak acoustic emission signals long before the uncertainty of the classification method reaches $10 \%$.
\end{abstract}

\title{
1 Introduction
}

Fiber reinforced composites show an extraordinary potential for application as light-weight structure materials due to their high strength-to-weight and high stiffness-to-weight ratio. Most of the time the investigation of material failure of fiber reinforced composites by conventional nondestructive techniques occurs offline, i.e. after loading and unloading of the specimen. In contrast, acoustic emission (AE) analysis is a powerful nondestructive technique for online monitoring of material failure during loading of the specimen [1]. Here, microscopic internal displacements like crack generation or crack propagation cause stress-waves that are detectable as transient acoustic waves. Within a platetype specimen, these acoustic waves are symmetric $\left(\mathrm{S}_{\mathrm{i}}\right)$ and antisymmetric $\left(\mathrm{A}_{\mathrm{i}}\right)$ Lamb-wave modes, as well as shear-horizontal $\left(\mathrm{SH}_{\mathrm{i}}\right)$ modes.

In addition to the activity of acoustic emission, the position of the acoustic emission source and the type of acoustic emission source are key aspects to enhance the understanding of material failure. While source localization in carbon fiber reinforced polymers (CFRP) still has to overcome the problem of anisotropic acoustic signal propagation and geometrical complexity [1-3], unsupervised pattern recognition techniques have already demonstrated their suitability to identify particular source mechanisms in CFRP [4-12].

The basis for these feature based pattern recognition approaches is the concept of feature extraction. Thus, typical features are calculated from the recorded acoustic 
emission signal (i.e. peak frequency, see also section 3) to parameterize the signal. Pattern recognition algorithms are then applied to search cluster structures in subsets of these features [13]. In general, the outcome of the pattern recognition process is a classification of acoustic emission signals based on their similarity to each other. The correlation of one group of acoustic emission signals to a particular source type is a separate task.

One problem common to all unsupervised pattern recognition approaches is the evaluation of the clustering result. For the case of acoustic emission analysis, the following two errors can occur:

1) A group of acoustic emission signals is assigned to the wrong source type

2) An acoustic emission signal is assigned to the wrong group

In literature, various methods are established to assign a group of AE signals to a particular source type [4-12]. In our previous publications [11,12,14-16] we use finite element modeling of acoustic emission signals for various source mechanisms validated in a variety of experimental configurations to perform this task.

However, a prerequisite for valid source identification is a statistically meaningful group of signals. If no distinction between the acoustic emission signals can be made based on their feature values, any subsequent discussion of the underlying source type is disputable. Since the exact assignment of one particular signal to one mechanism is by definition unknown, it is useful to express the error of the classification procedure as uncertainty of the assignment.

Within the present investigation we present a numerical method that is capable to quantify this uncertainty of assignment. We demonstrate how this method is used to calculate the uncertainty of assignment for an experimental dataset. We consider the influence of dispersion and attenuation effects during propagation of Lamb-waves on the extracted acoustic emission features and elaborate the experimental factors that cause an increase in the uncertainty of classification.

\section{Pattern recognition}

Since a comprehensive description of the pattern recognition method used in this investigation was previously reported in [11], we only give a brief summary in the following.

The presented method was inspired by the work of Anastassopoulos et al. and Günter et al. $[17,18]$ and is based on an exhaustive screening taking into account all combinations of signal features extracted from the recorded acoustic emission signals. For each possible combination of signal features an investigation of the classification performance of the k-means algorithm is evaluated ranging from two to ten classes. The numerical degree of cluster separation of each partition is calculated utilizing the Davies-Bouldin $(D B)$ and Tou ( TOU) indices, Rousseeuw's silhouette validation method $(S)$ and Hubert's Gamma statistics $(\gamma)$ [19-22]. Since the various cluster validation methods are comprehensively described in the authors' original work, their definition is not repeated in the following. 
In the spirit of $[17,18]$ the individual rating of each cluster validation technique is cumulated based on a voting scheme and is evaluated for the number of clusters with best performance. This is defined as the best partitioning for the given feature combination. This methodology can be used as an automated evaluation of the number of natural clusters and their partitions without previous knowledge about the cluster structure of acoustic emission signals.

The assignment of a group of acoustic emission signals to a source mechanism is achieved by a comparison to acoustic emission signals calculated by a finite element modeling approach. Since this is beyond the scope of the current investigation, we summarize the correlation between particular microscopic failure mechanisms and the respective acoustic emission source configuration briefly. The following correlation between micromechanical failure modes and acoustic emission source properties (notated in brackets) for fiber reinforced polymers is used:

- Interfiber fracture (matrix cracking or interfacial failure, in-plane)

- Fiber-Matrix Debonding (interfacial failure, in-plane and out-of-plane)

- Fiber-Matrix Pull-Out (interfacial failure, in-plane and out-of-plane)

- Interply delamination (matrix cracking or interfacial failure, out-of-plane)

- Fiber fracture (fiber breakage, in-plane)

A precise description of the implementation of the particular source types in finite element models is found in Ref. [15, 16]. The description of mesoscopic failure modes (e.g. fiber bridging) is beyond the scope of the proposed acoustic emission source models. The suitability of the proposed method for a variety of specimen geometries and loading conditions has been demonstrated using artificial, as well as experimental datasets $[11,12,14,15]$.

\subsection{Definition of the uncertainty of assignment}

As discussed in [11] the purpose of the pattern recognition technique is the detection of the natural clusters, which are defined as numerically best separation of the dataset investigated. However, the detection of natural clusters does not imply a classification error suitable for statistically significant identification of particular failure mechanisms in a material. Naturally, clusters will always have some overlap relative to each other, which causes ambiguous assignment of signals at the border between to clusters. Thus, a measure for the uncertainty of assignment during the classification process is required. In the following, the values of cluster validity measures $D B, T O U, S$ and $\gamma$ shall be used for this purpose.

In order to establish an analytical correlation between the cluster validity measures and the uncertainty of assignment we investigate artificially generated datasets. Following the approach of Milligan [23] and the refinement by Qiu and Joe [24] we generate datasets according to the implementation within the software package "R" by Qiu et al. [25]. We use the measure of the degree of separation $J$ as introduced by [26]. The measure $J$ is based on the separation of two clusters generated from two univariate normal distributions $\mathrm{N}(0,1)$ and $\mathrm{N}(0, \mathrm{~A})$ [26]. For the values of $\mathrm{A}=8, \mathrm{~A}=6$ and $\mathrm{A}=4$ the measure of the degree of separation ranges from "well-separated" $(J=0.342)$, "separated" $(J=0.213)$ to "close" $(J=$ 0.010 ) as shown in the scatter plots in figure 1. 
The proposed pattern recognition method can identify the number of clusters accurately down to $J=0.010$ [11]. Since the internal structure of the artificial datasets are initially known this allows a direct quantification of the misclassified cluster members of the test data sets.
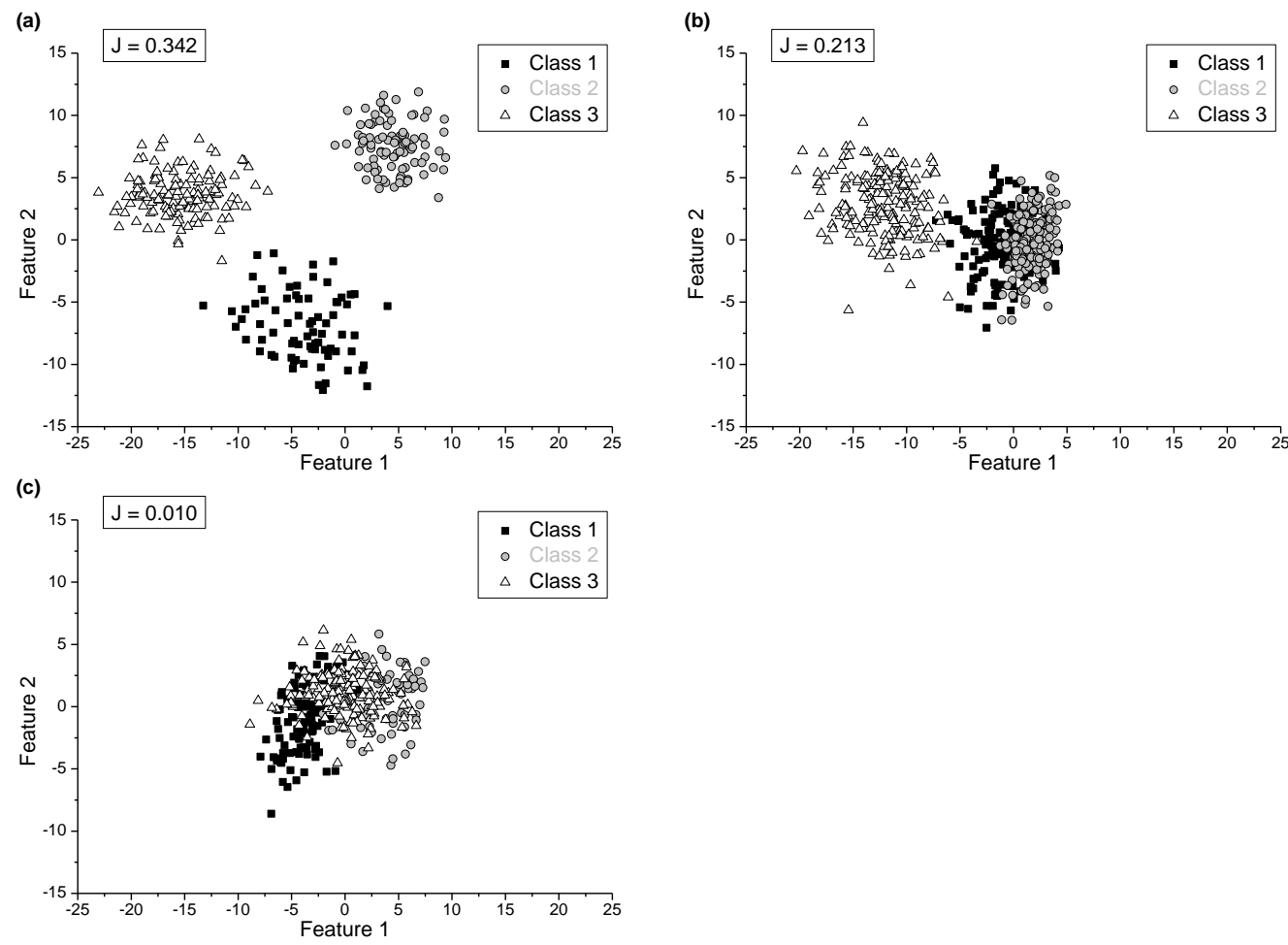

Figure 1: Visualization of cluster structure for degree of separation of $\mathbf{J}=0.342$ (a), $\mathrm{J}=0.213$ (b) and $\mathrm{J}=0.010(\mathrm{c})$.

As measure of the mismatch between two partitions the Rand index is applied [27]. In statistics, the Rand index is used as a direct measure of the percentage of decisions that are correct and thus is a direct measure of the classification error. Next we consider the correlation between the calculated cluster validity measures $D B, T O U, S$ and $\gamma$ and the Rand index.

To this end, we investigated a number of artificial datasets with varying degree of separation $J$ between -0.45 and 0.45 . The number of objects in each cluster was randomly chosen within the range [50,200] which reflects reasonable variation of the cluster sizes. Table 1 summarizes the remaining parameters used in the study. 


\begin{tabular}{|l|l|l|l|l|l|}
\hline Number of clusters & 2 & \multicolumn{3}{|c|}{3} & 5 \\
\hline Number of features & 5 & 4 & 5 & 6 & 5 \\
\hline Number of partitions generated & 16 & 22 & 105 & 22 & 16 \\
\hline
\end{tabular}

Table 1: Overview of settings used for generation of artificial datasets.

In the following we discuss the case of 3 clusters and 5 features to establish the correlation between the cluster validity measures and the Rand index.

Subsequently, we study the influence of the number of features and the number of clusters in sections 2.2 and 2.3 , respectively.

In figures $2 \mathrm{a}-2 \mathrm{~d}$ values of the Rand index are plotted as a function of the four cluster validity measures investigated. Since all cluster validity measures are by definition linked to the quality of the partition a correlation to the Rand index is expected. Here, $D B$ indicates a partition of high quality by a numerical minimum, while TOU, $S$ and $\gamma$ maximize their values for high cluster separation.

The scatter in figures $2 \mathrm{a}-2 \mathrm{~d}$ indicates that there is no direct analytical correlation between a particular cluster validity measure and the Rand index. This is owed to the statistical distribution of clusters elements, which can cause nearly identical values of $D B, T O U, S$ or $\gamma$ for two partitions, while the respective number of correct classifications is still different for both partitions.

We use a five-parameter logistic function to fit the cluster validity measure $c$ to the Rand index. This was found to yield the best fit of the data points among the class of sigmoidal growth functions.

$$
R A N D=A_{\min }+\frac{A_{\max }-A_{\min }}{\left(1+\left(\frac{c}{c_{0}}\right)^{-h}\right)^{s}}
$$

The boundary conditions of the Rand index with lower limit of $A_{\min }=0$ and upper limit of $A_{\max }=1$ reduce the number of fit parameters by two. The resulting fit including the $95 \%$ prediction band is shown in figures $2 \mathrm{a}-2 \mathrm{~d}$. Fit parameters $c_{0}$, h and $\mathrm{s}$ are shown in the respective inset. 
(a)

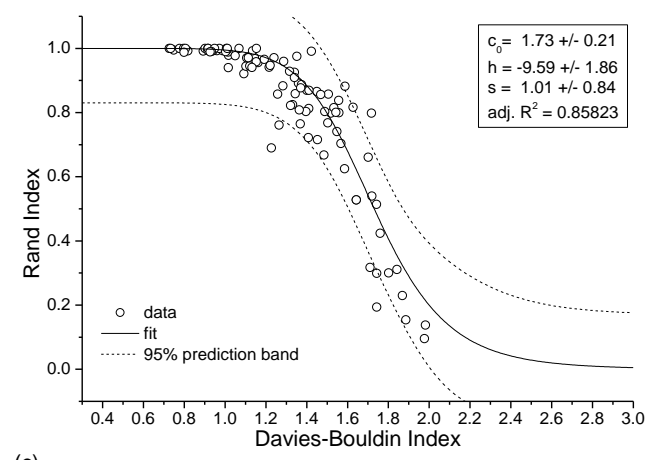

(c)

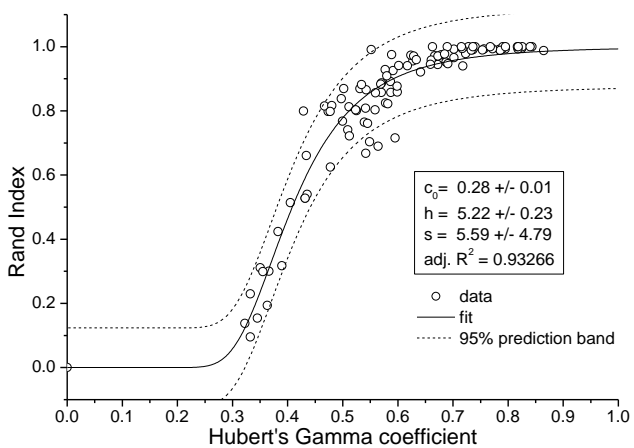

(b)

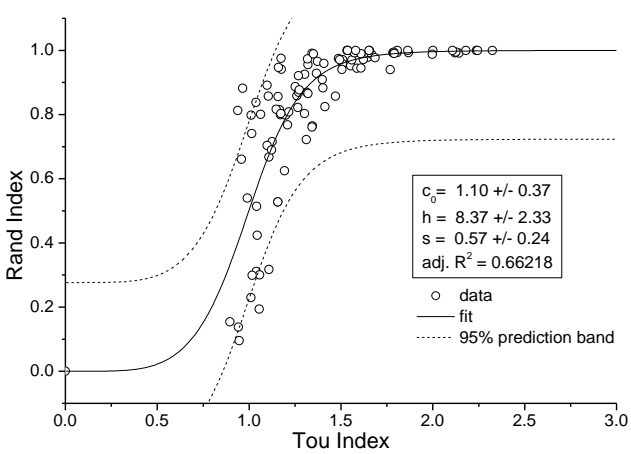

(d)

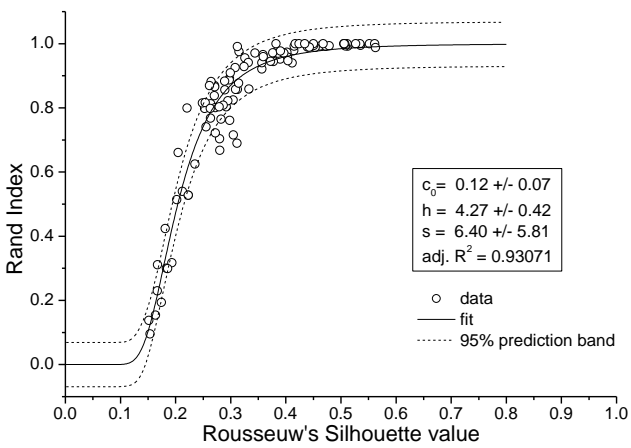

Figure 2: Rand Index as a function of cluster validity statistics. Plots include results of fit model and their corresponding 95\% prediction band. Diagrams are shown for Davies-Bouldin index (a), Tou index (b), Hubert's Gamma coefficient (c) and Rousseuw's Silhouette value (d).

As indicated by the values of the adjusted least square errors $\left(\operatorname{adj} . R^{2}\right)$, the various cluster validity measures show different performance in their correlation to the Rand index value. Best performance was observed for $S$ and $\gamma$. A strong correlation was found for $D B$ while the size of the $95 \%$ prediction band for TOU was found to be insufficient for further usage with an experimental data set. The reason for the different performance of the cluster validity measures is their different sensitivity to outliers and statistical scatter within the datasets $[11,13$, 18]. Here, $S$ and $\gamma$ show less sensitivity to such effects, translating into less scatter of their numerical values. The value of $D B$ is affected more and the value of $T O U$ suffers drastically from outliers and minor changes in the dataset.

In the following subsections we will discuss the influence of the number of features and the number of clusters using exemplarily the fit parameters for the values of $S$ only.

\subsubsection{Influence of number of features}

Next we investigate the influence of the number of features selected for the classification process on the fit parameters of equation (1). We vary the number of features between four and six keeping the number of clusters constant at three. As seen from figure 3 for Rousseuw's silhouette value $S$, there is a significant impact on the fit parameters when the number of features is varied. The same impact was also observed on the remaining cluster validity measures $D B, T O U$ and $\gamma$. Since the fit parameters for the cases with four, five and six features are not identical within their error margins an individual set of fit parameters is recommended for each number of features. Visually this is expressed by the different slopes and shifts of the fit functions in figure 3 . 


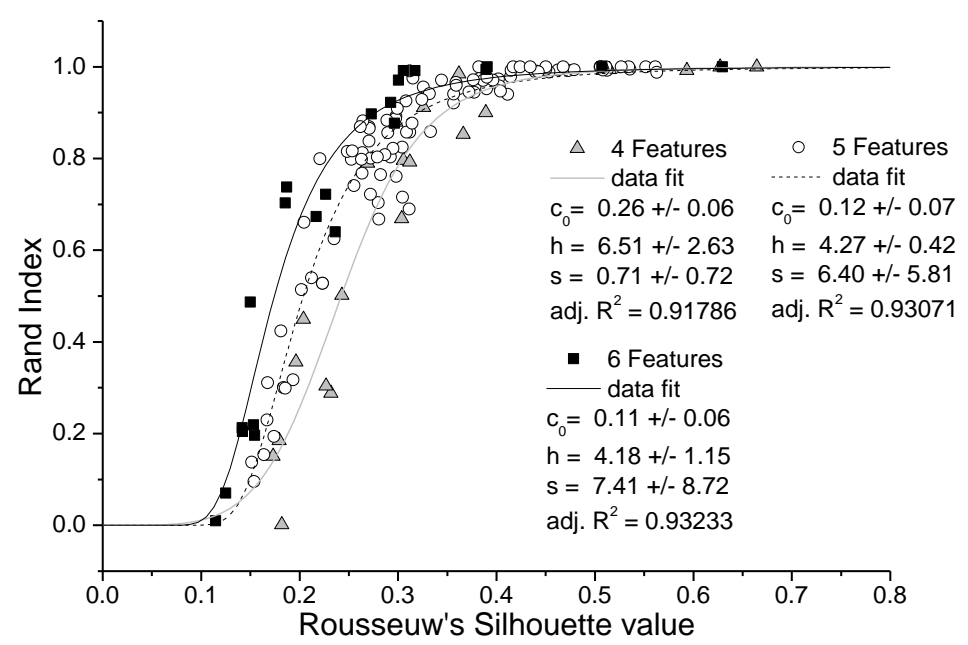

Figure 3: Rand Index as a function of Rousseuw's Silhouette value for artificial partitions with four, five and six features including results of fit model.

\subsubsection{Influence of number of clusters}

In analogy to the investigation in section 2.2 we now study the influence of the number of clusters. To this end, we vary the number of clusters between two and five, keeping the number of features constant as five. The result of the investigation is shown in figure 4 for Rousseuw's silhouette value $S$. Within the margin of error the values of the fit parameters are identical. Similar behavior was also observed for the remaining cluster validity measures $D B, T O U$ and $\gamma$. This indicates, that the number of clusters is not a relevant quantity for the correlation between the chosen cluster validity measure and the Rand index. This behavior can be expected, since all cluster validity measures investigated are by definition independent of the number of clusters (see original work [19-22]).

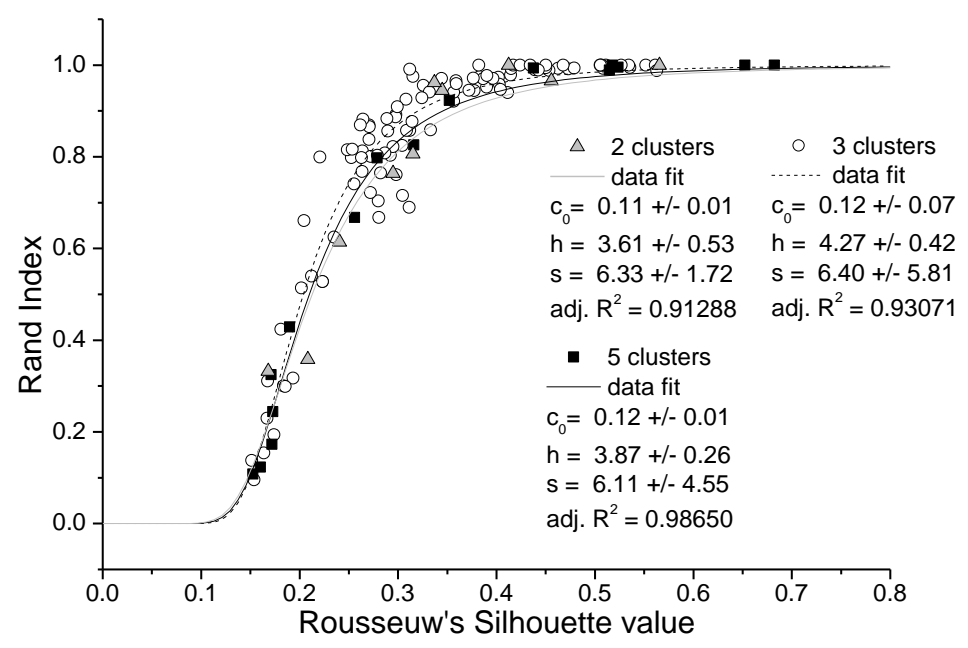

Figure 4: Rand Index as a function of Rousseuw's Silhouette value for artificial partitions with 3 and 5 clusters including results of fit model. 


\subsection{Calculating the uncertainty of assignment for an experimental dataset}

Up to this point artificial datasets were used to establish a correlation between the Rand index and cluster validity measures. The calculation of the first requires a reference partition with the known assignment of each signal to the respective cluster. Since this assignment is unknown for experimental datasets, a direct application of the Rand index is not possible. Instead, it is possible to calculate the cluster validity indices for any partition of an experimental dataset. Based on the outcome of the previous sections we use only the values of $D B, S$ and $\gamma$. The value of TOU will not be used further in the investigation, since the adjusted least square error of the fit procedure from section 2.1 was not found to be sufficient.

In the first step we calculate the cluster validity indices for the partition of the experimental dataset. The corresponding Rand index is then calculated from equation (1) using the fit parameters for the number of features used in the experimental dataset. The calculated value of the Rand index is then defined as uncertainty of assignment. For an improved interpretability, we calculate the mean value of all Rand index values obtained for the individual cluster validity measures. This is further used as uncertainty of assignment.

Since the algorithm to relate the Rand index and a cluster validity measure $\mathrm{c}$ is based on the structure of the artificial datasets, the experimental datasets must have similar structure to yield correct values for the uncertainty of assignment. One possibility to investigate if the experimental dataset is reasonable close to the artificial dataset is a comparison of their feature distributions as shown in figure 5 . The figure 5-a shows the univariate distribution of one of the features of an artificial dataset with 3 clusters as generated by the approach of Qiu and Joe [2426]. This is now compared to the feature distribution of the experimental datasets as used in the following sections. Many times, experimentally used features span only certain intervals (e.g. frequency ranges, percentages, etc.) and have upper limits or lower limits. Before a comparison of the dataset structure is made, it is thus suitable to remove those limitations by performing a normalization of the feature values followed by a principle components analysis [13]. It is worth noting, that the latter does not change the cluster validity measures, but can alter the visual appearance of the feature distribution range significantly. Therefore we compare the distribution of the first principle component feature in figure 5-b to the feature distribution of the artificial dataset in figure 5-a. Both show an univariate distribution in reasonably close agreement to each other. This comparison is made for each of the features. If those also show sufficient agreement, the structure of the datasets is considered to be similar enough to yield appropriate values for the uncertainty of assignment. 
(a)

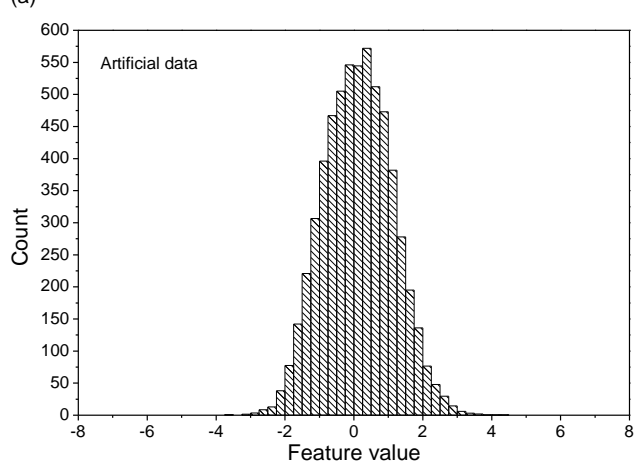

(b)

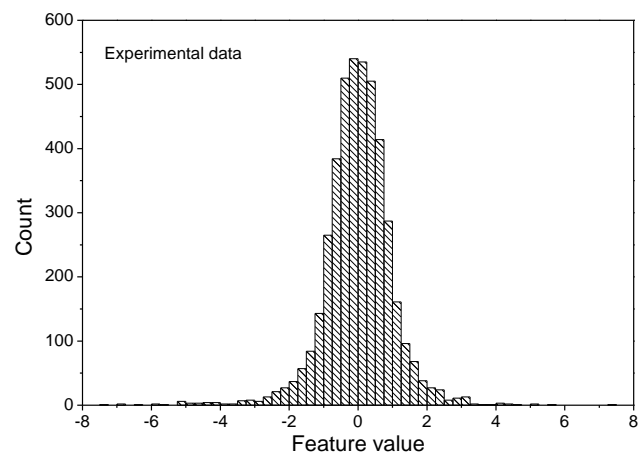

Figure 5: Univariate feature distribution for first feature of an artificial dataset with 3 clusters (a) and feature distribution of first principal component feature of experimental dataset (b).

\section{Experimental}

In this section, we describe the experimental setup to obtain acoustic emission signals from testing of double cantilever beam (DCB) specimens. The specimens investigated are made from the prepreg system Sigratex CE 1250-230-39. The curing cycle follows the standard procedure recommended by the material supplier. All six specimens were prepared with unidirectional stacking sequence and outer dimensions of $570 \mathrm{~mm} \times 40 \mathrm{~mm} \times 6.4 \mathrm{~mm}$ (length $\times$ width $\times$ thickness) as seen in figure 6 . These dimensions are substantially larger than commonly used standards like e.g. ASTM D 5528. Here, the goal is the generation of acoustic emission sources within a well-controlled distance to the detecting sensor. The chosen specimen geometry with $570 \mathrm{~mm}$ length is more than two times larger than the typical size of $125 \mathrm{~mm}$ to $250 \mathrm{~mm}$ length and thus provides a crack propagation length of approximately $275 \mathrm{~mm}$. For load introduction, two aluminum blocks of $80 \mathrm{~mm} \times 40 \mathrm{~mm} \times 19 \mathrm{~mm}$ (length $\times$ width $\times$ thickness) were glued to the specimen using Stycast 2850. All experiments were carried out on a Zwick 1464 spindle driven machine with constant crosshead-speed of 5.0 $\mathrm{mm} / \mathrm{min}$.

For detection of acoustic emission signals, two multi-resonant sensors (model WD) were mounted on the specimen using suction cup holders. To provide suitable acoustic transmission, medium viscosity silicone paste (type Baysilone) was used. All signals were detected using a PCI-2 acquisition system with $40 \mathrm{~dB}$ preamplification, $35 \mathrm{~dB}$ threshold level, $10 \mathrm{MSP} / \mathrm{s}$ sampling rate and 10/80/300 (Peak-definition-time/Hit-definition-time/Hit-lockout-time) trigger settings. Bandpass settings were chosen to be $20 \mathrm{kHz}$ to $3 \mathrm{MHz}$. All acoustic emission signals were localized using a localization algorithm based on the arrival time difference of the acoustic emission signals. Since the progress of damage in the DCB specimen will cause splitting of the specimen into two beams, the Lambwaves propagating in the split beam will be different from those propagating in the remaining part of the specimen. To analyze the accuracy of the localization procedure we performed ten load-hold cycles during one experiment and conducted pencil lead breaks (PLBs) in the damaged state at each hold cycle. The localization results did not show any significant deviation to the source positions found in the undamaged state.

For investigation by pattern recognition, only localized signals of the sensor mounted on the undamaged part of the beam were taken into account. The features extracted from the signals are summarized in table 2 . It is worth noting, 
that the features in table 2 are not meant to provide a comprehensive list for characterization of acoustic emission signals. The selected features are based on the conclusions of previous investigations $[15,16]$ and are considered suitable for further analysis by our pattern recognition approach. Their definition is based on the basic properties derived from the signal in time domain $U(t)$ and in frequency domain $\widetilde{U}(f) . N_{A E}$ are the number of threshold crossings in time domain, $t_{A E}$ is the duration of the signal. The features $N_{\text {peak }}$ and $t_{\text {peak }}$ refer to the number of threshold crossings and the time of the maximum signal amplitude, respectively. The feature $f_{\text {peak }}$ defines the frequency position of maximum intensity, while the definition of $f_{\text {centroid }}$ is given in table 2. The definition of the different Partial Power features is noted in table 2 as well.

\begin{tabular}{|c|c|}
\hline AE feature & Definition \\
\hline Average Frequency $[\mathrm{kHz}]$ & $\langle f\rangle=N_{A E} / t_{A E}$ \\
\hline Reverbaration Frequency [kHz] & $f_{\text {rev }}=\frac{N_{A E}-N_{\text {peak }}}{t_{A E}-t_{\text {peak }}}$ \\
\hline Initiation Frequency [kHz] & $f_{\text {init }}=N_{\text {peak }} / t_{\text {peak }}$ \\
\hline Peak-Frequency [kHz] & $f_{\text {peak }}$ \\
\hline Frequency centroid [kHz] & $f_{\text {centroid }}=\frac{\int f \cdot \tilde{U}(f) d f}{\int \widetilde{U}(f) d f}$ \\
\hline Weighted Peak-Frequency [kHz] & $\left\langle f_{\text {peak }}\right\rangle=\sqrt{f_{\text {peak }} \cdot f_{\text {centroid }}}$ \\
\hline Partial Power $1[\%]$ & $\int_{f_{1}}^{f_{2}} \widetilde{U}^{2}(f) d f$ \\
\hline Partial Power $2[\%]$ & \\
\hline Partial Power 3 [\%] & PP1: f1 $=0 \mathrm{kHz} ; \mathrm{f} 2=150 \mathrm{kHz}$ \\
\hline Partial Power $4[\%]$ & $\begin{array}{l}\text { PP3: } \mathrm{f} 1=300 \mathrm{kHz} ; \mathrm{f} 2=450 \mathrm{kHz}\end{array}$ \\
\hline Partial Power 5 [\%] & PP4: f1 $=450 \mathrm{kHz} ; \mathrm{f} 2=600 \mathrm{kHz}$ \\
\hline Partial Power 6 [\%] & PP6: $\mathrm{f} 1=900 \mathrm{kHz} ; \mathrm{f} 2=1200 \mathrm{kHz}$ \\
\hline
\end{tabular}

Table 2. Definition of AE features.

In addition to mechanical loading, one specimen was investigated using acoustic emission test sources as seen in figure 6. Pencil lead breaks were applied at the top and the edge of the plate at different source-sensor distances. Also, one WD sensor was used as piezoelectric pulser to generate test signals at different sourcesensor distances. These test sources cause three characteristic types of test signals as shown in the Choi-Williams distributions in figures 7-a to 7-c for a sourcesensor distance of $280 \mathrm{~mm}$ [28]. To identify the individual wave modes, we added superimposed Lamb-wave dispersion curves in figures 7-a to 7-c calculated for the density and elastic coefficients $\mathrm{C}_{\mathrm{ij}}$ as given in table 3 and plate thicknesses of $6.4 \mathrm{~mm}$ and $40 \mathrm{~mm}$.

\begin{tabular}{|l|l|}
\hline Material property & Value \\
\hline Density & $1550\left[\mathrm{~kg} / \mathrm{m}^{3}\right]$ \\
\hline $\mathrm{C}_{11}$ & $133.5[\mathrm{GPa}]$ \\
\hline $\mathrm{C}_{12}=\mathrm{C}_{13}$ & $7.3[\mathrm{GPa}]$ \\
\hline $\mathrm{C}_{22}=\mathrm{C}_{33}$ & $12.9[\mathrm{GPa}]$ \\
\hline $\mathrm{C}_{23}$ & $8.8[\mathrm{GPa}]$ \\
\hline $\mathrm{C}_{44}$ & $2.1[\mathrm{GPa}]$ \\
\hline $\mathrm{C}_{55}=\mathrm{C}_{66}$ & $6.1[\mathrm{GPa}]$ \\
\hline
\end{tabular}

Table 3. Material properties used for dispersion curve calculation. 
Pencil lead breaks at the top of the plate generate strong $\mathrm{A}_{0}$-modes as shown in figure 7-a. Due to the intensity of the $\mathrm{A}_{0}$-mode the initial part of the signal is not resolved well in the Choi-Williams distribution. A decrease of the coefficient scale by a factor 1000 reveals the initial part of the signal and allows identification of the $S_{0}$-mode and the $S_{2}$-mode in the beginning of the signal.

In contrast, the pencil lead break at the side surface of the plate excites a $\mathrm{S}_{2}$-mode in the frequency range between $220 \mathrm{kHz}$ and $400 \mathrm{kHz}$ as seen in figure 7-b. The contribution in the Choi-Williams distribution at $120 \mathrm{kHz}$ does not fall into the range of the Lamb-wave modes calculated for the $6.4 \mathrm{~mm}$ plate thickness. The contribution observed at $120 \mathrm{kHz}$ propagates faster than the respective antisymmetric modes and exhibits frequency content, which is below those calculated for the $\mathrm{S}_{0}$-mode. One possible explanation for this contribution is the propagation of a $S_{2}$-mode excited along the $40 \mathrm{~mm}$ thick y-direction of the specimen. The frequency position and arrival time is in good agreement with the dispersion curve calculated for a plate thickness of $40 \mathrm{~mm}$.

Finally, the signal of the WD pulser shown in figure 7-c exhibits a combination of a strong $\mathrm{S}_{2}$-mode, superimposed by a weaker $\mathrm{S}_{0}$-mode and an $\mathrm{A}_{2}$-mode.

Using these three types of test sources with distinctly different modal composition and frequency ranges, it is possible to investigate the signal propagation of different Lamb-wave modes in this specimen geometry experimentally.

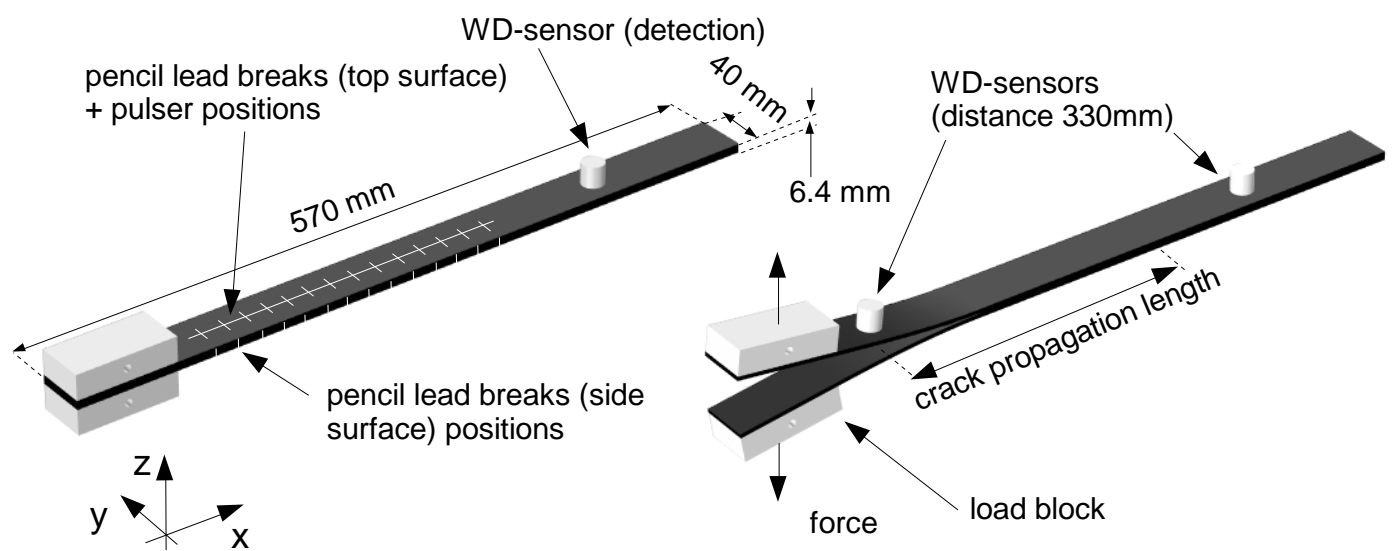

Figure 6: Scheme of experimental setup used for generation of AE test sources (left) and for fracture mechanics experiment (right). 
(a)

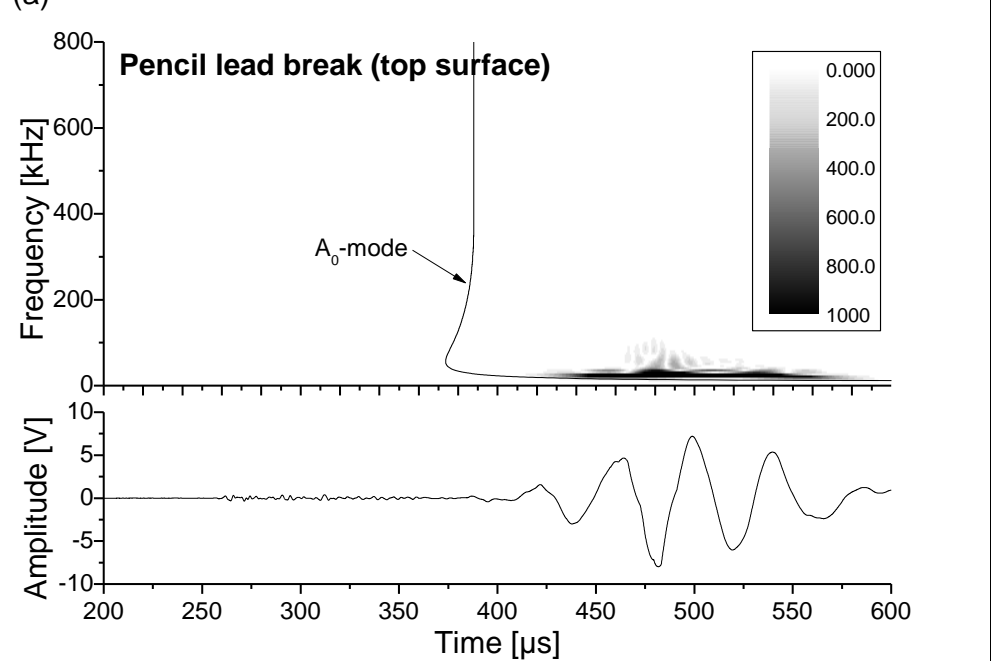

(b)

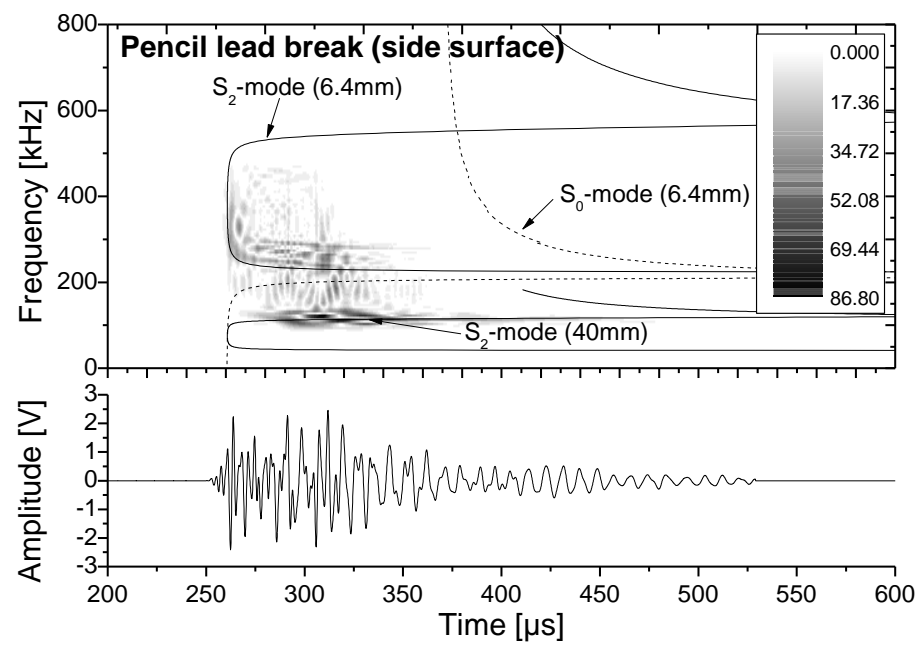

(c)
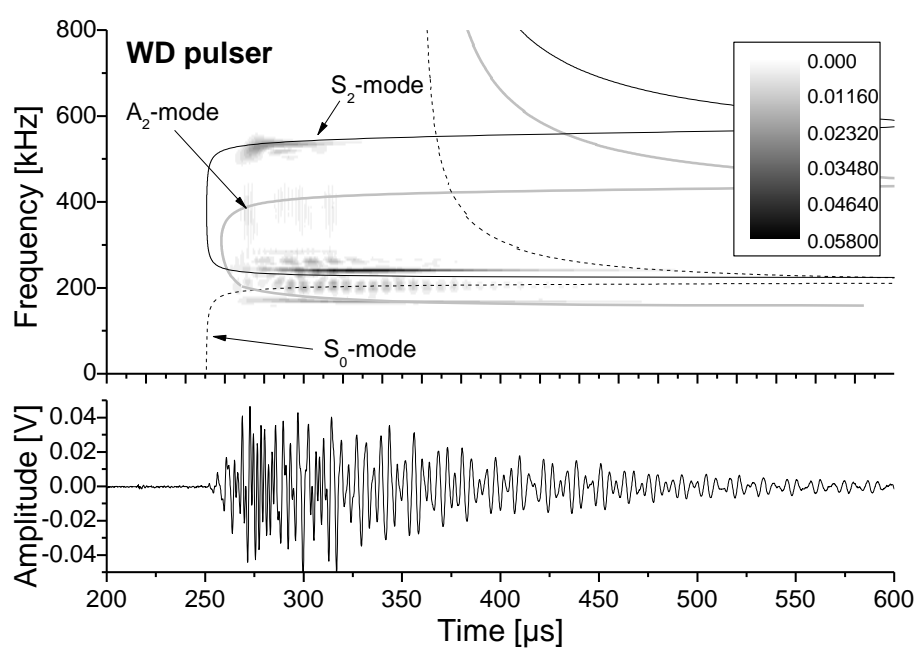

Figure 7: AE signals of different acoustic emission test sources evaluated at $280 \mathrm{~mm}$ source-sensor distance. Graphs show Choi-Williams distribution for signal of top surface pencil lead break (a), side surface pencil lead break (b) and WD-pulser (c). 


\section{Results and Discussion}

In this section, we apply the algorithm developed in section 2 to calculate the uncertainty of assignment of our pattern recognition approach [11] in application to experimental data. We discuss the origin of the obtained clusters in terms of the modal composition of the acoustic emission signals. The impact of the sourcesensor distance on the uncertainty of assignment is elaborated. In the last subsection we relate our findings to the influence of attenuation as measured in our specimens.

\subsection{Relation between cluster structure and source-sensor distance}

We applied the pattern recognition method presented in [11] to the experimental data obtained from testing of large double cantilever beam specimens. Analogous to our previous investigations on double cantilever beam specimens with dimensions in accordance with ASTM D 5528 [12] the pattern recognition approach is able to detect three distinct clusters. From all the features of table 2 the features Partial Power 1, Partial Power 2, Partial Power 4, Peak-Frequency and Weighted Peak-Frequency were selected by the algorithm described in [12] to yield the natural clusters, as shown in figure 8 . Based on this previous investigation and conclusions from other previous work we attribute the three clusters to the occurrence of matrix cracking, interfacial failure and fiber breakage as marked in figure $8[11,12,14-16]$.

As explained in section 3, three types of acoustic emission test sources were applied at source-sensor distances between $80 \mathrm{~mm}$ and $280 \mathrm{~mm}$. The signal features Partial Power 2 and Weighted Peak-Frequency were calculated from the obtained test signals and their data points are superimposed in figure 8 to those from double cantilever beam testing.

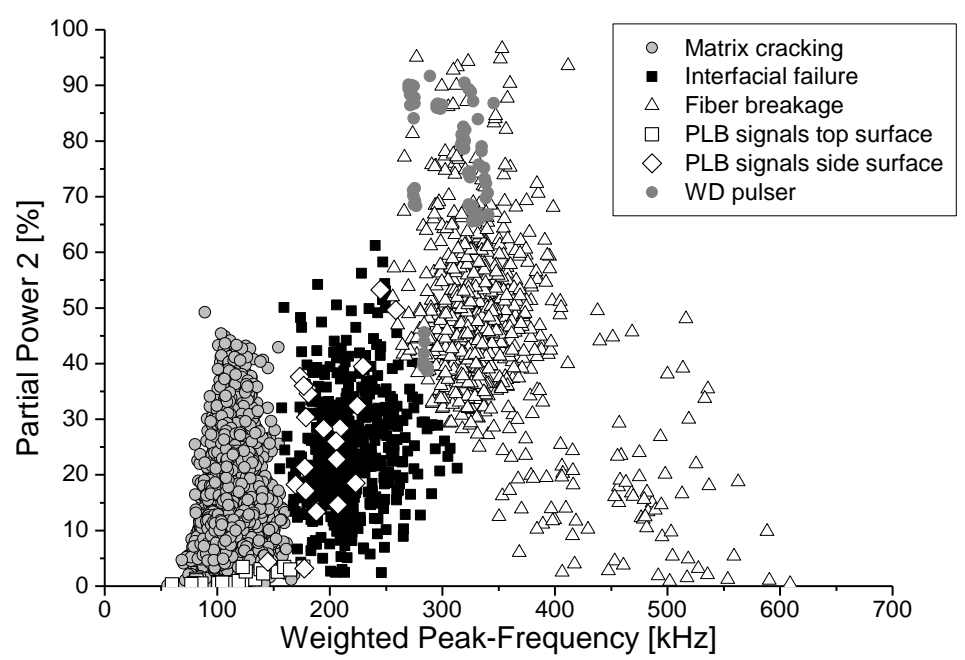

Figure 8: Comparison of position of signal features from $\mathrm{AE}$ test sources and measurement data from DCB test.

As mentioned in section 3, each of the three acoustic emission test sources excites a unique combination of symmetric and antisymmetric modes. This translates into a unique feature range as seen in figure 8. 
It has been shown in [16], that there are at least three parameters that influence the ratio of Lamb-wave modes and shear-horizontal modes present in acoustic emission signals:

1) The source microstructure (e.g. the source type)

2) The depth position of the source within the plate

3) The distance between the source and the point of detection

There is a remarkable coincidence of the feature range of the Weighted PeakFrequency of the pencil lead breaks at the top surface with the cluster attributed to matrix cracking. Since the depth position of these pencil lead breaks is constant and the source type is identical, this feature range is solely attributed to the change in source-sensor distance. As demonstrated by FEM-simulations of acoustic emission signals of matrix cracking [14-16], changes in the depth of the source position can also cover the full range of Partial Power 2 up to $50 \%$ as observed for the experimental signal features. Since matrix cracking is expected to occur not only located at the medial plane of the plate but also inside the beams such changes in the depth of the source positions are expected.

The feature range of signals of pencil lead breaks at the edge of the plate coincides well with the cluster attributed to interfacial failure. Here, both feature ranges (Partial Power 2 and Weighted Peak-Frequency) are well reflected by the signals detected at different source-sensor distances. Since interfacial failure is expected to occur mostly at the medial plane of the specimen, this behavior is also in agreement with the experimental observation. In addition to the effect of sourcesensor distance, there is a natural variability to the microstructure of acoustic emission source types correlated to interfacial failure. This is likely to cause the additional extension of the cluster related to interfacial failure as seen in figure 8 .

For the signals attributed to fiber breakage, part of the associated cluster is covered by the feature values of signals from the WD pulser. Here the change in source-sensor distance causes a significant shift in the feature values Partial Power 2 and Weighted Peak-Frequency. However, the position of the WD pulser at the surface of the specimen is not identical to the depth position of fiber breakage. The latter is expected to occur dominantly within the specimen at the positions of high bending moments. The respective change in depth position towards the medial plane of the specimen would translate into higher contributions of symmetric modes, which would cause higher values of Weighted Peak-Frequency and lower values of Partial Power 2. This effect has also been demonstrated by FEM-simulations of fiber breakage in previous work [14-16].

We want to point out, that we do not suggest using pencil lead breaks as test sources to emulate matrix cracking or piezoelectric pulser to emulate fiber breakage. Instead, those test sources should be used to excite distinct ratios of Lamb-wave modes as described in section 3. If those are representative for a particular failure mechanism will strictly depend on their position, the specimen geometry and stacking sequence.

One consequence of these observations is that the size of the clusters and their overlap will depend on the distance between source and sensor. This is owed to dispersion and attenuation effects during signal propagation. The influence of 
source-sensor distance on the extracted features was validated experimentally by signals of an identical test source. Naturally, the overlap of clusters will govern the uncertainty of the assignment of an acoustic emission signal to a particular failure mechanism. Thus we investigate the change in the cluster validity measures for different source-sensor distances to obtain the respective uncertainty of assignment.

\subsection{Uncertainty of assignment as function of source-sensor distance}

In order to evaluate the source-sensor distance for the loading of the double cantilever beam specimens, the $\mathrm{x}$-position of the acoustic emission signals was localized in the geometry according to figure 6 . The density of source positions was calculated using the software program Density Ville [29]. In figure 9 the $x-$ position is plotted as gray-scale density diagram as a function of time for one representative specimen. Superimposed is the measured force-time curve of the respective experiment. Clearly, the majority of the acoustic emission signals in double cantilever beam testing originate from positions close to the crack tip. Only few signals are localized significantly ahead of or behind the position of the crack tip. The density diagram also reveals that the crack progress is not completely continuous, but consists of subsequent jumps of the crack front. Using the localized acoustic emission source position, the distance to the detecting sensor can be calculated.

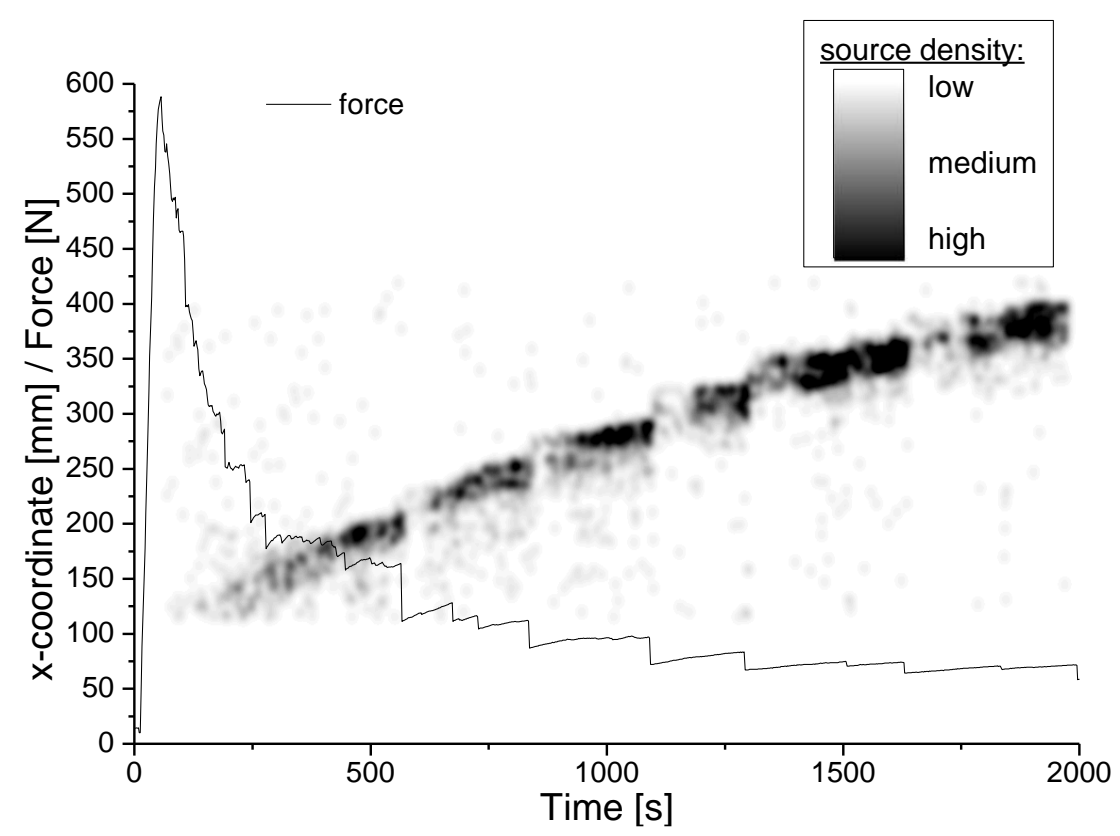

Figure 9: Force time curve and AE source density for one representative specimen.

In the following, we distinguish between two approaches to investigate the influence of the source-sensor distance on the uncertainty of the assignment. As shown in figure 10, the experimental datasets were evaluated based on their source-sensor distance. As first approach the signals were analyzed cumulatively. Thus, the first subset of the dataset is localized close to the sensor and has a small width distribution of source positions. The next subset of the dataset is farther away and has an increased width distribution of source positions. The subsets are chosen in steps of 500 signals until the full dataset size is reached. Thus, for each specimen around 3500 to 4000 subsets of the complete dataset were investigated. 
As second approach the subsets of the dataset are chosen to be of equal size of 500 signals. Their mean $\mathrm{x}$-position is shifted for each subset until the maximum distance to the sensor is reached. In this configuration around 3000 to 3500 subsets of the complete dataset were investigated for each specimen.
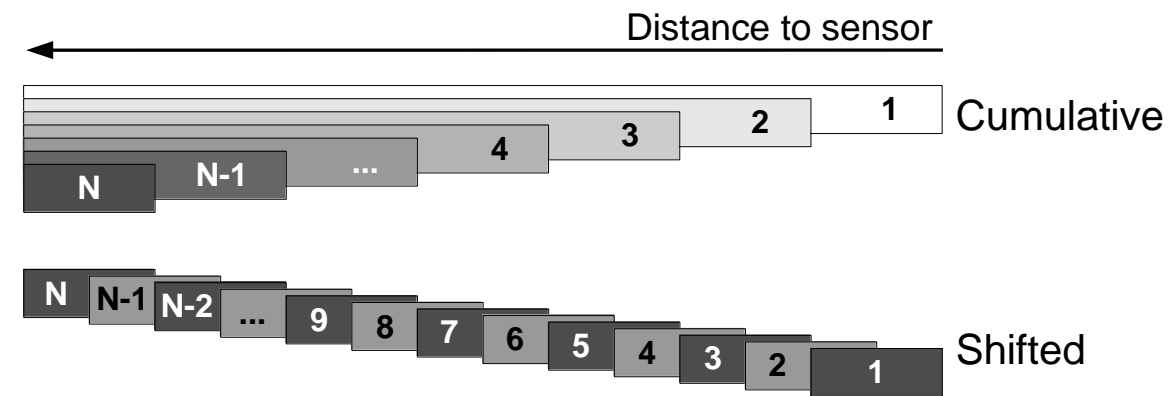

Figure 10: Scheme for calculation of cluster validity indices as function of source-sensor distance.

For each subset of the whole dataset, the Davies-Bouldin Index, Rousseuw's Silhouette value and Hubert's Gamma coefficient are calculated based on the features Partial Power 1, Partial Power 2, Partial Power 4, Peak-Frequency and Weighted Peak-Frequency as defined in table 2. The result for both approaches are shown for one representative specimen in figure 11-a and 11-b, respectively. Using equation (1) and the fit parameters of figure 2, the uncertainty of assignment can be calculated for each of the cluster validity measures $D B, S$ and $\gamma$ according to the description of section 2.2. The result is shown in figure 11-c and $11-\mathrm{d}$ for the shifted and cumulative approach, respectively.

(a)

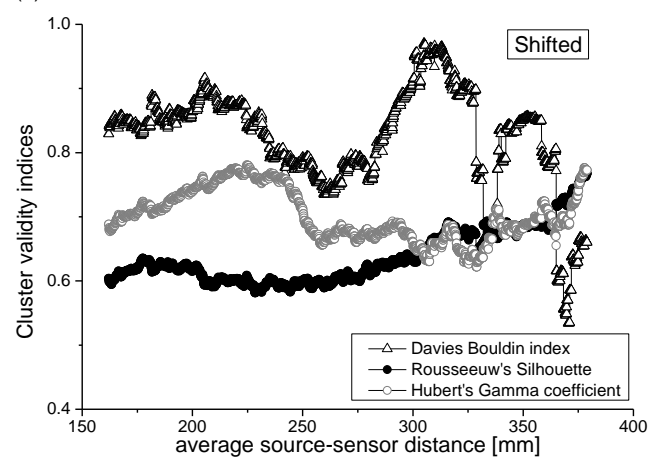

(c)

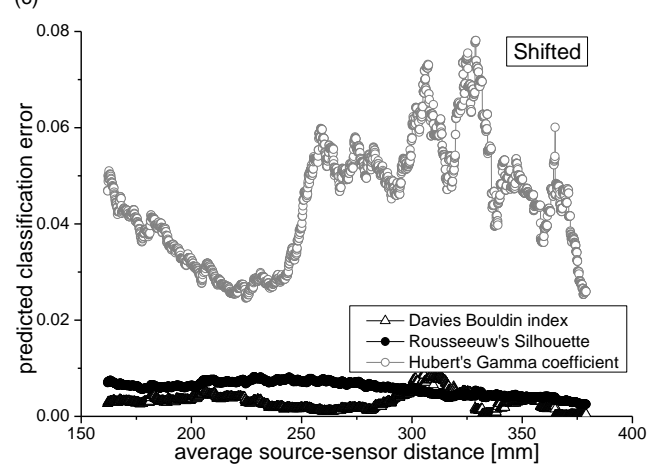

(b)

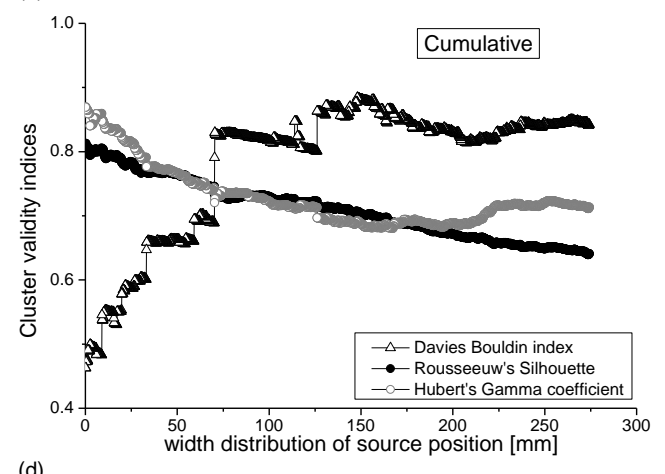

(d)

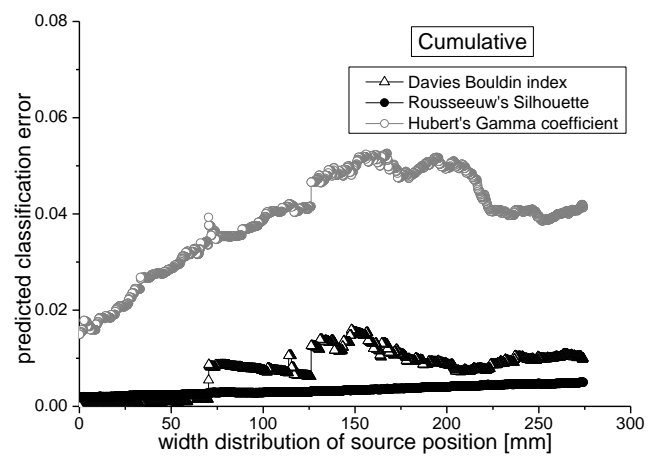

Figure 11: Calculation of cluster validity indices as function of distance for shifted (a) and cumulative (b) approach and their respective uncertainty of assignment for shifted (c) and cumulative (d) approach. 
For the shifted approach, no clear trend of the cluster validity measures or their respective uncertainty of assignment is observed as a function of the sourcesensor distance. This leads to the conclusion, that the source-sensor distance below $375 \mathrm{~mm}$ does not have a critical impact on the uncertainty of assignment of one acoustic emission signal to a particular cluster.

In contrast, the cumulative approach reveals an increased uncertainty of assignment with increasing width distribution of the source positions. Here, the width distribution of sources within the specimen seems to influence the accuracy of the source identification procedure significantly. This effect was found in all six double cantilever beam specimens. The respective findings are summarized in table 4 . The values of the uncertainty of assignment based on the cluster validity measures $D B, S$ and $\gamma$ are reported for the width distribution below $140 \mathrm{~mm}$ and below $275 \mathrm{~mm}$ only. In addition, their mean value is given. It was found, that an increasing width of source distributions causes an higher uncertainty of assignment in all specimens. Their individual values range from $1.6 \%$ to $7.1 \%$.

\begin{tabular}{|c|l|l|l|l|l|l|l|l|}
\hline & \multicolumn{4}{|l|}{$\begin{array}{l}\text { uncertainty of assignment (width of } \\
\text { source distribution }<140 \mathrm{~mm} \text { ) }\end{array}$} & \multicolumn{3}{l|}{$\begin{array}{l}\text { Uncertainty of assignment (width of } \\
\text { source distribution < 275mm) }\end{array}$} \\
\hline specimen & DB & S & G & MEAN & DB & S & G & MEAN \\
\hline A & 0.073 & 0.032 & 0.083 & 0.064 & 0.100 & 0.039 & 0.068 & 0.069 \\
\hline B & 0.036 & 0.006 & 0.023 & 0.022 & 0.019 & 0.010 & 0.058 & 0.029 \\
\hline C & 0.045 & 0.009 & 0.068 & 0.040 & 0.066 & 0.015 & 0.131 & 0.071 \\
\hline D & 0.015 & 0.007 & 0.056 & 0.026 & 0.019 & 0.010 & 0.055 & 0.028 \\
\hline E & 0.012 & 0.009 & 0.038 & 0.020 & 0.027 & 0.010 & 0.062 & 0.033 \\
\hline F & 0.020 & 0.007 & 0.020 & 0.016 & 0.025 & 0.013 & 0.030 & 0.023 \\
\hline
\end{tabular}

Table 4. Uncertainty of assignment for width of source distribution $<140 \mathrm{~mm}$ and width of source distribution $<275 \mathrm{~mm}$.

\subsection{Influence of attenuation}

In order to investigate the influence of attenuation we applied pencil lead breaks at source-sensor distances between $80 \mathrm{~mm}$ and $280 \mathrm{~mm}$ at the top of the specimen as shown in figure 6 . The signal amplitude in $\mathrm{dB}_{\mathrm{AE}}(\mathrm{dB}$ amplitude corrected by preamplification factor and gain settings) is shown in figure 12. Since the equipment used has a saturation level of $97 \mathrm{~dB}_{\mathrm{AE}}$ the amplitudes of the $\mathrm{A}_{0}$ mode measured at $80 \mathrm{~mm}$ distance were not taken into account for linear regression. The attenuation is evaluated as $-0.165 \pm 0.008 \mathrm{~dB} / \mathrm{mm}$ for the $\mathrm{A}_{0}$-mode and -0.047 $\pm 0.005 \mathrm{~dB} / \mathrm{mm}$ for the $S_{0}$-mode, respectively. Since the attenuation measurement is in the near-field of the source, the current value is attributed to geometric spreading [30,31]. It is worth noting, that an extrapolation to larger distances has to consider the different contributions of geometric spreading, thermoelastic dissipation, modal and frequency dispersion during signal propagation [30,31]. Since those have distinctly different contributions as function of propagation distance, a linear extrapolation of the current values can only be considered as worst case. 


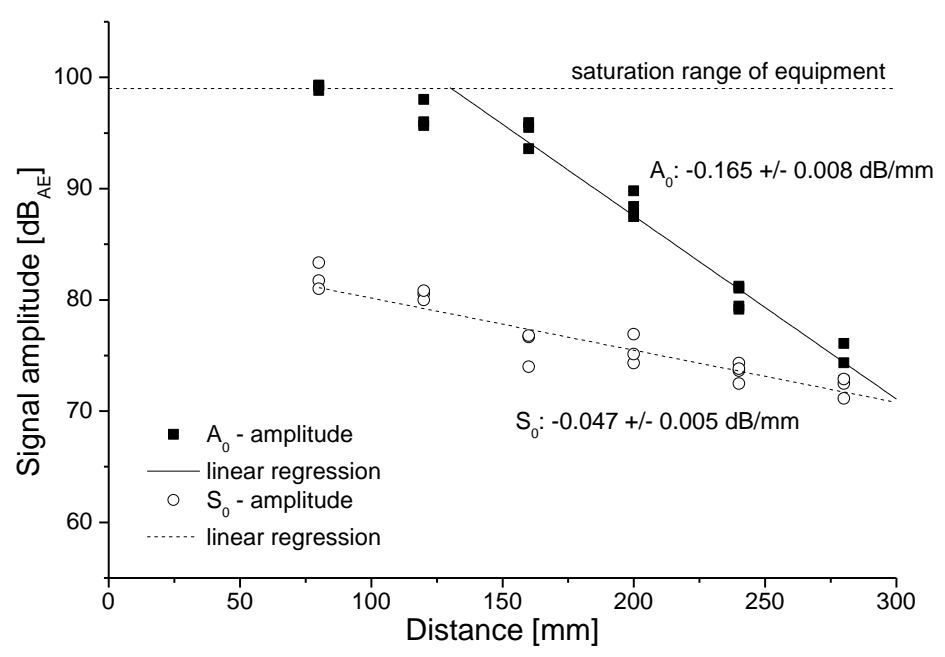

Figure 12: Attenuation measurement of fundamental Lamb-wave modes in unidirectional CFRP using pencil lead breaks at the top of the plate. Measurement of $\mathrm{A}_{0}$-mode at $80 \mathrm{~mm}$ was not taken into account for linear regression.

Generally, for a typical threshold level between $25 \mathrm{~dB}_{\mathrm{AE}}$ and $35 \mathrm{~dB}_{\mathrm{AE}}$ and saturation range between $100 \mathrm{~dB}_{\mathrm{AE}}$ and $120 \mathrm{~dB}_{\mathrm{AE}}$ the dynamic measurement range available lies within $65 \mathrm{~dB}_{\mathrm{AE}}$ and $95 \mathrm{~dB}_{\mathrm{AE}}$. Using the attenuation level of -0.165 $\mathrm{dB} / \mathrm{mm}$ of the $\mathrm{A}_{0}$-mode this translates into a distance between $393 \mathrm{~mm}$ and $575 \mathrm{~mm}$ before the incident signal falls below the detection threshold. These distances are only valid for an acoustic emission source with high signal strength, i.e. between $100 \mathrm{~dB}_{\mathrm{AE}}$ and $120 \mathrm{~dB}_{\mathrm{AE}}$. The majority of acoustic emission signals originating from material failure in carbon fiber reinforced plastics is typically between $50 \mathrm{~dB}_{\mathrm{AE}}$ and $60 \mathrm{~dB}_{\mathrm{AE}}$ and may fall below the threshold level already at distances less than $151 \mathrm{~mm}$.

\section{Conclusion}

Using the relation between cluster validity measures and Rand index values we established an approach to estimate the uncertainty of assignment of a pattern recognition result.

Since the approach presented is based on artificial datasets, we would like to point out the two requirements for direct application of the proposed method:

1) The structure of the experimental dataset has to be compatible with the structure of the artificial datasets.

2) The number of features and number of clusters has to be identical to those used to obtain the associated fit parameters.

If the cluster structure does not coincide with the artificial dataset, the reported fit parameters will only yield an approximation. However, the method can still be applied if artificial datasets are generated that have a similar structure as the experimental dataset.

If the above requirements are fulfilled, the presented approach allows quantification of the uncertainty of assignment for any pattern recognition approach or other classification method applied to any experimental dataset. That way, this approach can be used as a tool for the community to assess the statistical 
significance of any assignment of the experimental dataset before considering further steps of validation.

In the current investigation the application of the method was demonstrated using acoustic emission signals of large double cantilever beam specimens. Applying an established pattern recognition method, we were able to distinguish between three types of signals associated with matrix cracking, interfacial failure and fiber breakage. The uncertainty of the assignment to the respective clusters was calculated by equation (1) using the fit parameters of figure 2 and the cluster validity measures of the experimental dataset partition.

The formation of clusters in feature space and their relation to the underlying Lamb-wave modes was investigated. Using pencil lead breaks and a piezoelectric pulser on the top and pencil lead breaks on the side surface of the specimen we were able to investigate the influence of the source-sensor distance experimentally. It was demonstrated that part of the distribution in feature space is solely caused by the varying source-sensor distance. Other contributions arise from the source microstructure or the depth position of the source.

We were able to quantify the uncertainty of assignment of the partitions of the experimental datasets as a function of source-sensor distance. It turned out, that the source-sensor distance is not a critical parameter for the accuracy of source identification. Instead, the width distribution of the source positions was found to cause an increased uncertainty of assignment. Based on the calculated uncertainty of assignment for source-sensor distances below $375 \mathrm{~mm}$ we conclude that such source-sensor distances still allows meaningful source identification procedures.

Further, we found that the maximal source-sensor distance for low amplitude signals in CFRP may be limited to a range even below $115 \mathrm{~mm}$. For larger distances it is likely, that some of the acoustic emission signals fall below the detection threshold. Similar, for high amplitude signals we estimate the maximal source-sensor distance to be in the range up to $575 \mathrm{~mm}$.

Based on these findings we conclude that the limiting factor for meaningful pattern recognition approaches will be the acoustic attenuation rather than the increase in overlap of the cluster structure due to increased source-sensor distances.

\section{Acknowledgments}

We would like to thank Marvin A. Hamstad for providing the dispersion curve results used in this publication.

\section{References}

[1] Große C, Ohtsu M (2008) Acoustic emission testing. Springer, Berlin Heidelberg

[2] Li J, Qi G (2009) Improving Source Location Accuracy of Acoustic Emission in Complicated Structures. J. Nondest. Eval. 28:1-8 doi: 10.1007/s10921-009-0042-z 
[3] Wang L, Yuan FG (2007) Group velocity and characteristic wave curves of Lamb waves in composites: Modeling and experiments. Comp. Sci. Technol. 67:1370-1384 doi:

10.1016/j.compscitech.2006.09.023

[4] Huguet S, Godin N, Gaertner R, Salmon L, Villard D (2002) Use of acoustic emission to identify damage modes in glass fibre reinforced polyester. Comp. Sci. Technol. 62:1433-1444 doi: $10.1016 / \mathrm{S} 0266-3538(02) 00087-8$

[5] Ramirez-Jimenez CR, Papadakis N, Reynolds N, Gan T, Purnell P, Pharaoh M (2004) Identification of failure modes in glass/polypropylene composites by means of the primary frequency content of the acoustic emission event. Comp. Sci. Technol. 64:1819-1827 doi: 10.1016/j.compscitech.2004.01.008

[6] Marec A, Thomas JH, Guerjouma R (2008) Damage characterization of polymer-based composite materials: Multivariable analysis and wavelet transform for clustering acoustic emission data. Mech. Syst. Signal Process., 22:1441-1464 doi: 10.1016/j.ymssp.2007.11.029

[7] Li X, Ramirez C, Hines EL, Leeson MS, Purnell P, Pharaoh M (2008) Pattern Recognition of Fiber-reinforced Plastic Failure Mechanism using Computational Intelligence Techniques. Neural Networks, pages 2340-2345. IEEE World Congress on Computational Intelligence.

[8] Philippidis T, Nikolaidis V, Anastassopoulos A (1998) Damage Characterisation of C/C laminates using Neural Network Techniques on AE signals. NDT\&E International 31:329-340 doi: 10.1016/S0963-8695(98)00015-2

[9] Ativitavas N, Pothisiri T, Fowler TJ (2006) Identification of Fiber-reinforced Plastic Failure Mechanisms from Acoustic Emission Data using Neural Networks. J. Comp. Mat. 40:193-226 doi: $10.1177 / 0021998305053458$

[10] Kostopoulos V, Karapappas P, Loutas T, Vavouliotis A, Paipetis A, Tsotra P (2011) Interlaminar Fracture Toughness of Carbon Fibre-Reinforced Polymer Laminates With Nano- and Micro-Fillers. Strain 47:269-282 doi: 10.1111/j.1475-1305.2008.00612.x

[11] Sause MGR, Gribov A, Unwin AR, Horn S (2012) Pattern recognition approach to identify natural clusters of acoustic emission signals. Pattern Recognition Let. 33:17-23 doi: 10.1016/j.patrec.2011.09.018

[12] Sause MGR, Müller T, Horoschenkoff A, Horn S (2012) Quantification of failure mechanisms in mode-I loading of fiber reinforced plastics utilizing acoustic emission analysis. Comp. Sci. Technol. 72:167-174 doi: 10.1016/j.compscitech.2011.10.013

[13] Polikar R (2006) Pattern Recognition. In: Wiley Encyclopedia of Biomedical Engineering, chapter Pattern Recognition. John Wiley \& Sons, Inc., New York, 2006.

[14] Sause MGR, Horn S (2010) Influence of specimen geometry on acoustic emission signals in fiber reinforced composites: FEM-simulations and experiments. In: Conf. Proc. 29th European Conf. on Acoustic Emission Testing, Vienna, Austria

[15] Sause MGR (2010) Identification of failure mechanisms in hybrid materials utilizing pattern recognition techniques applied to acoustic emission signals. Dissertation, Augsburg University

[16] Sause MGR, Horn S (2010) Simulation of acoustic emission in planar carbon fiber reinforced plastic specimens. J. Nondest. Eval. 29:123-142 doi: 10.1007/s10921-010-0071-7

[17] Anastassopoulos AA, Philippidis TP (1995) Clustering methodology for the evaluation of acoustic emission from composites. J. Acoust. Emission 13:11-21

[18] Günter S, Bunke H (2003) Validation indices for graph clustering. Pattern Recognition Lett. 24:1107-1113 doi: 10.1016/S0167-8655(02)00257-X

[19] Davies DL, Bouldin DW (1979). A cluster separation measure. IEEE Trans. Pattern 
Anal. Mach. Intell. 1:224-227

[20] Tou JT (1979) DYNOC - a dynamic optimal cluster-seeking technique. Int. J. Comput. Inform. Sci. 8-541-547.

[21] Rousseeuw PJ (1987) Silhouettes: a graphical aid to the interpretation and validation of cluster analysis. J. Comput. Appl. Math. 20:53-65

[22] Hubert LJ, Arabie P (1985) Comparing partitions. J. Classif. 2:193-218

[23] Milligan GW (1985) An Algorithm for Generating Artificial Test Clusters, Psychometrika, 50:123-127

[24] Qiu WL, Joe H (2006) Generation of Random Clusters with Specified Degree of Separation. J. Classification, 23:315-334 doi: 10.1007/s00357-006-0018-y

[25] Qiu WL, Joe H (2009) clusterGeneration: random cluster generation (with specified degree of separation), 2009

[26] Qiu WL, Joe H (2006) Separation Index and Partial Membership for Clustering. Computational Statistics and Data Analysis, 50:585-603 doi: 10.1016/j.csda.2004.09.009

[27] Rand WM (1971) Objective criteria for the evaluation of clustering methods. Journal of the American Statistical Association 336:846-850 doi: 10.2307/2284239

[28] Choi H-I, Williams JW (1989) Improved Time-Frequency Representation of Multicomponent Signals Using Exponential Kernels. IEEE Transactions on Acoustics, Speech and Signal Processing 37:862-871 doi: 10.1109/ASSP.1989.28057

[29] Skopalik W, Sause MGR (2012) DensityVille Manual Rev. 0.99 http://www.physik.uniaugsburg.de/exp2/downloads/. Accessed 01 August 2012

[30] Pollock AA (1990) Critical AE Problems for the Researcher. Journal of Acoustic Emission 9:140-141

[31] Downs KS, Hamstad MA (1995) Correlation of Regions of Acoustic Emission Activity with Burst Locations for Spherical Graphite/Epoxy Pressure Vessels. Journal of Acoustic Emission 13:56-66 